\title{
Typical Imaging Findings of Renal Oncocytoma
}

\author{
Bhumika Dua, Rajaram Sharma*, Tapendra N. Tiwari, Saurabh Goyal \\ Department of Radio-Diagnosis, Pacific Institute of Medical Sciences, Umarda, Udaipur, Rajasthan, India
}

Article Info

\section{Article Notes}

Received: November 09, 2021

Accepted: January 27, 2022

\section{*Correspondence:}

${ }^{*}$ Dr. Rajaram Sharma, Department of Radio-Diagnosis

Pacific Institute of Medical Sciences, Umarda, Udaipur

Rajasthan, India; Email: hemantgalaria13@gmail.com

${ }^{\circ} 2022$ Sharma R. This article is distributed under the terms of the Creative Commons Attribution 4.0 International License.

\section{Keywords}

Renal tumors

Radiology

Urology

\section{ABSTRACT}

For the past few decades, a solid mass in the kidney with avid enhancement was considered renal cell carcinoma (RCC). With the advancement in radiological interventions, the understanding and treatment of a large number of tumours has changed. Oncocytomas are solid benign renal masses contributing $3-7 \%$ in all renal neoplasms. We report a case of a 32-year-old male who presented to our medicine OPD with abdominal pain and discomfort for about one month, later diagnosed with oncocytoma. We emphasize the importance of the typical imaging findings for the diagnosis and characterization of renal tumors.

\section{Description}

For the past few decades, a solid mass in the kidney with avid enhancement was considered renal cell carcinoma (RCC). With the advancement in radiological interventions, the understanding and treatment of a large number of tumours has changed ${ }^{1}$. Oncocytomas are solid benign renal masses contributing 3-7\% in all renal neoplasms. There is a high prevalence in men, with the peak incidence in the seventh decade of life. Most of the people having oncocytoma are asymptomatic. When the size of the mass increases, the patient may present with flank pain or abdominal mass $^{2}$. Oncocytomas and chromophobe RCCs are often referred to as oncocytic neoplasms due to the same origin ${ }^{3}$. They have overlapping features with RCC, which makes it difficult to diagnose correctly. The presence of a central stellate scar, commonly referred to as spoke wheel pattern (the peripheral rim of vessels from which centripetal vessels converge centrally); is potentially helpful in making the diagnosis, but this scar is present in only about one-third of the cases and may also be present in RCCs. The presence of metastasis or aggressive infiltration into the surrounding structures is a reliable feature usually found in $\mathrm{RCCs}^{4}$. Another distinguishing feature for oncocytomas is the highest amount of contrast washout amongst the renal tumours, followed by clear cell RCC, chromophobe RCC and papillary RCC ${ }^{5}$. Also, the percentage of arterial enhancement in oncocytomas is greater than $500 \%$. Due to non-differentiation from RCCs, these are usually excised. Although, oncocytoma, if proven, may not require surgery unless the size becomes large and causes unpleasant symptoms.

A 32-year-old male presented to our medicine OPD with abdominal pain and discomfort for about one month. He was advised ultrasound (USG) whole abdomen. On USG, there was a lobulated hypoechoic mass present on the lower pole of the right kidney [Figure 1A]. On Colour Doppler, the mass showed peripheral vascularity and certain small branches of vessels 
converging inwards, giving the typical spoke wheel appearance (the vessels converging centrally) [Figure 1B]. Differential diagnoses of renal masses, RCC or oncocytoma was given. The probability of oncocytoma was considered because of no significant symptoms or other positive findings on abdominal USG. He was further advised contrast-enhanced computerized tomography (CECT) and magnetic resonance imaging (MRI) abdomen for further characterization and status of perirenal fascia. On unenhanced CT, there was a well-defined, round to oval, isodense mass with the central hypodense area, measuring approx. $33 \times 37 \times 28 \mathrm{~mm}$; on the anterior part of the lower pole of the right kidney [Figure 2A]. On post-contrast CT scan, there was a spoke wheel pattern of vascularity and delayed enhancement of central scar. The mass showed significant peripheral enhancement $(\sim 110 \mathrm{HU})$ and washout $(\sim 65 \mathrm{HU})$ on cortical and equilibrium phases, respectively [Figure $2 \mathrm{~B} \& 2 \mathrm{C}$ ]. There was displacement of renal vessels and duodenum but no obvious invasion into surrounding structures [Figure 2D]. On MRI, the mass appeared well defined. It was hypointense on T1 weighted images and hyperintense on T2 weighted images. Also, there was no obvious breach in perirenal fascia or infiltration in perinephric space [Figure $3 \mathrm{~A}$ and $3 \mathrm{~B}]$. On the basis of clinical picture and radiological investigations, the diagnosis of oncocytoma was given. The patient has been kept on the follow up and till now there is no change in the tumor size or morphology.
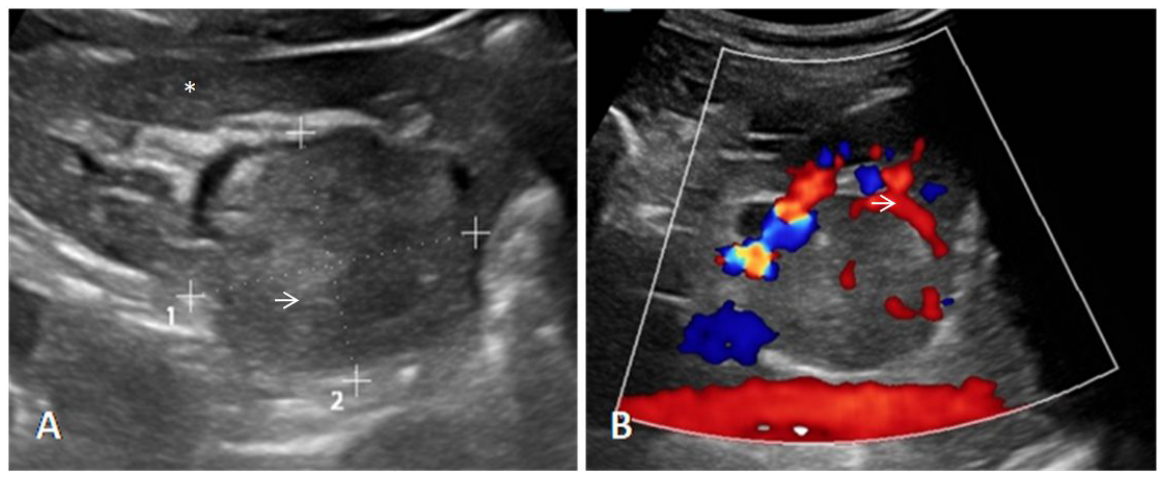

Figure 1: Trans abdomen ultrasonography (A) Image shows a round to oval hypoechoic mass (white arrow) on the lower pole of the right kidney (asterixis). (B) Colour Doppler ultrasound image, the mass shows a peripheral rim of vessels (white arrow) with centripetal radiating vessels forming spoke wheel pattern.

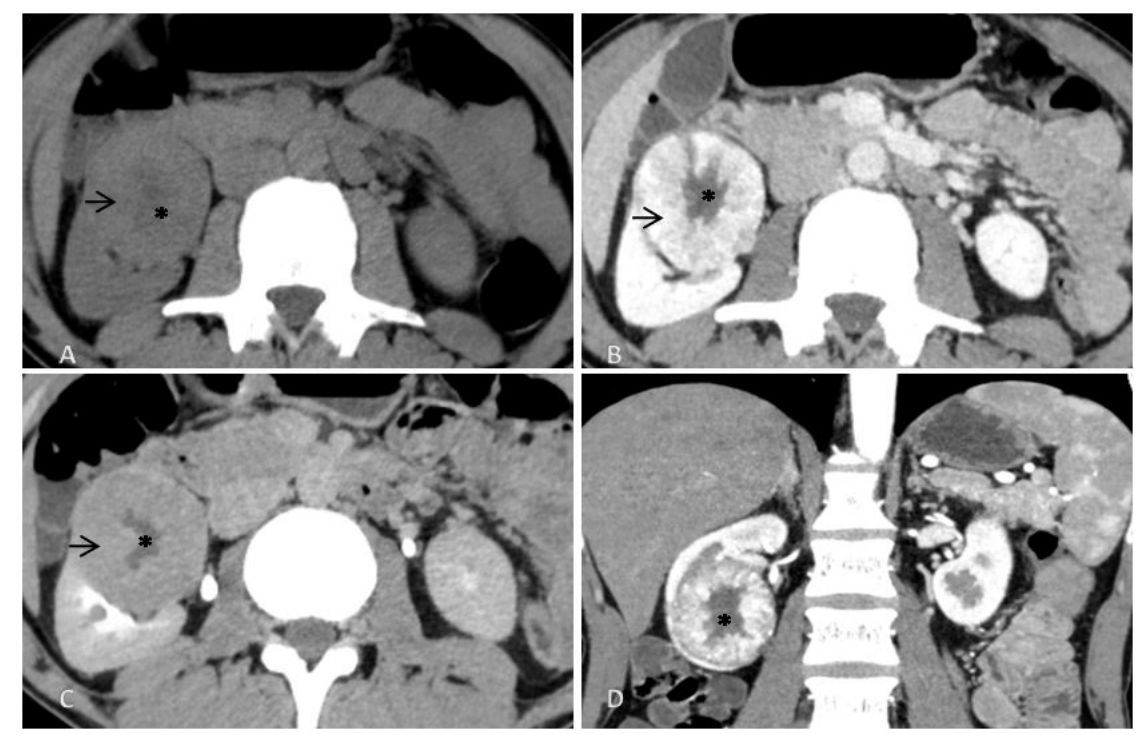

Figure 2: Non-contrast and contrast-enhanced axial and coronal sections of computerized tomography of the abdomen. (A) Axial section from non-contrast CT abdomen showing a well-defined, round to oval, isodense mass lesion with a central hypodense area (asterixis) on the anterior aspect of the lower pole of the right kidney. (B) Axial section from the cortical phase of contrast-enhanced CT abdomen showing avid peripheral enhancement of the mass (black arrow) with central non enhancing central hypodense stellate scar (asterixis) forming classical spoke wheel pattern. (C) Axial section from equilibrium phase of contrast-enhanced CT abdomen showing significant washout of contrast from the peripheral rim (black arrow). (D) Coronal section from the cortical phase of contrast-enhanced CT abdomen showing no definite signs of perinephric fat stranding or invasion of surrounding structures. 

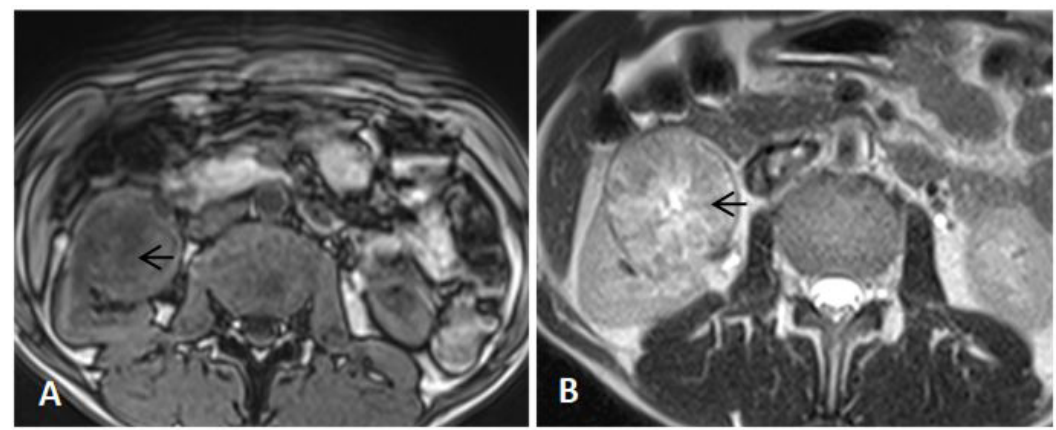

Figure 3: Unenhanced MRI abdomen (A \& B) Axial sections from T1 and T2 weighted images from MRI abdomen shows a well-circumscribed abnormal signal intensity lesion on the anterior aspect of the lower pole of the right kidney. It appears hypointense on T1 weighted images and predominantly hyperintense on $\mathrm{T} 2$ weighted images. There is no obvious sign of pararenal fascia involvement or infiltration of surrounding structures.

\section{Learning Points}

- Benign renal tumours such as oncocytoma are usually asymptomatic and detected incidentally. These may present with vague flank pain.

- Characteristic findings of oncocytoma on imaging helps to differentiate from other aggressive tumours of the kidney include a well-defined mass with a central scar, characteristic spoke wheel appearance and intense arterial enhancement and absence of any perinephric or vascular invasion.

- Categorizing renal masses into benign and malignant on imaging is useful in avoiding unnecessary interventions and helps in preoperative planning.

\section{References}

1. Allen $B C$, Tirman $P$, Jennings Clingan $M$, et al. Characterizing solid renal neoplasms with MRI in adults. Abdom Imaging. 2014; 39(2): 358-387.

2. Bostwick DG, Cheng L. Urologic surgical pathology. Mosby Inc. (2008) ISBN:0323019706.

3. Rosenkrantz $A B$, Hindman $N$, Fitzgerald $E F$, et al. MRI features of renal oncocytoma and chromophobe renal cell carcinoma. AJR Am J Roentgenol. 2010; 195(6): W421-W427.

4. Galmiche C, Bernhard JC, Yacoub M, et al. Is multiparametric MRI useful for differentiating oncocytomas from chromophobe renal cell carcinomas? AJR Am J Roentgenol. 2017; 208(2): 343-350.

5. Bird VG, Kanagarajah P, Morillo G, et al. Differentiation of oncocytoma and renal cell carcinoma in small renal masses $(<4 \mathrm{~cm})$ : the role of 4-phase computerized tomography. World J Urol. 2011; 29(6): 787-792. 